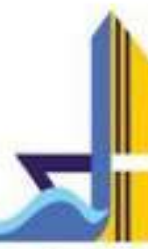

Université de Damiette

Faculté des Lettres

Département de Français

\title{
Analyse comparative de la BD "Persepolis" de Marjane Satrapi et de son adaptation filmique
}

Préparée par

Basma Ibrahim Mohamed

Assistante à la faculté de pédagogie Département des langues étrangères

Université de Mansoura

$\underline{\text { Sous la direction de }}$

Dr. Racha Mahmoud EL-KHAMISSY

Professeur de linguistique à la faculté Al-Alsun

Université d'Ain-Chams

\section{Dr. Hani Georges FANOUS}

Professeur adjoint de linguistique à la faculté des lettres

Université de Damiette

$$
\text { ro }
$$




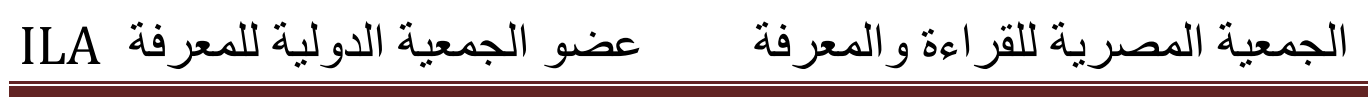




\section{الجمعية المصرية للقر اعة والمعرفة عضو الجمعية الدولية للمعرفة}

\section{Introduction}

Persepolis est une série de $\mathrm{BD}$ en noir et blanc de la dessinatrice iranienne Marjane Satrapi. Cette série se compose de quatre albums qui ont été publiés entre les années 2000 et 2003. La narratrice y raconte son autobiographie depuis son enfance à Téhéran après la chute du Chah de l'Iran et pendant la révolution islamiste jusqu'à son entrée difficile dans la vie adulte en Europe. Marjane Satrapi est née en 1969 dans une famille libérale et démocratique en Iran. Elle a étudié dans une école française à Téhéran. En 1984, elle est envoyée, après la révolution, par ses parents à Vienne en Autriche pour échapper au nouveau régime islamiste. Elle a continué ses études au lycée français de Vienne, puis elle est retournée en Iran afin de suivre des études supérieures. En 1994, elle est partie en France pour chercher sa liberté et pour faire des études sur le dessin et l'art. Elle réside actuellement à Paris où elle travaille comme dessinatrice.

En 2007, Marjane Satrapi a adapté ses albums au cinéma avec le cinéaste français Vincent Paronnaud. L'histoire de Persepolis, qui représente un portrait de la société iranienne pendant 16 ans, de 1978 jusqu'à 1994, met l'accent sur l'évolution de la vie de l'écrivaine comme un long voyage de la servitude à la liberté. En fait, nous avons ressenti l'intérêt d'analyser la BD et l'adaptation filmique de Persepolis parce que c'est un type artistique totalement différent. Persepolis se caractérise, grâce à Marjane Satrapi, par sa richesse des sentiments féminins opposés entre la servitude et la liberté qui présentent une période censurée et critique dans l'histoire politique de l'Iran.

L'adaptation filmique ou "le roman à l'écran" est l'opération de la transformation de l'œuvre littéraire à un film cinématographique, 
c'est la transformation du mot à l'image. En réalité, adapter un texte littéraire au cinéma n'est pas simple: c'est une tâche très compliquée, puisqu'il faut d'abord avoir un scénario inspiré du texte original qui sera valable à l'adaptation car tout récit écrit n'est pas nécessairement convenable à l'adaptation. Raison pour laquelle le cinéaste ou bien le scénariste doit mettre le texte adapté sous une forme d'écriture audiovisuelle.

Certes, la réussite de l'adaptation est souvent marquée par l'excès affectif qu'elle ajoute au texte original. Depuis 1958, le critique français André Bazin a réfléchi sur la problématique de l'adaptation cinématographique en ce qui concerne la fidélité ou la trahison du roman d'origine dans les tomes de la collection du septième art. C'est lui qui a ouvert le débat de la fidélité à la lettre ou à l'esprit de l'œuvre originale. Pour cela, l'adaptation est deux types: l'adaptation fidèle et l'adaptation libre.

Dans l'adaptation cinématographique, la fidélité est de respecter le contexte, les actions, l'ordre des événements racontés, les caractéristiques des personnages et leurs fonctions dans le récit. Bref, c'est l'adaptation littérale.

Quant à l'adaptation libre, elle est une œuvre séparée du texte original parce que le cinéaste s'inspire du thème majeur de la source littéraire originale puis il compte sur son imagination et sa fiction pour l'accomplissement de son film. En conséquence, cette adaptation exige un bon réalisateur qui peut habilement construire un bon film. D'une manière générale, les spectateurs préfèrent ce type de l'adaptation qui incite à la recréation dans le monde du cinéma.

Le choix définitif de prendre tel ou tel type d'adaptation est toujours lié à la nature de la source littéraire, à sa possibilité de 
transformation en film et au point de vue du cinéaste. Dans l'adaptation filmique Persepolis, Marjane Satrapi a mélangé les deux types en restant parfois fidèle mais aussi en faisant beaucoup de changements dans les actions par la suppression de certains détails.

Sans aucun doute, la lecture des quatre albums de Persepolis nous a aidés beaucoup dans la compréhension de l'adaptation filmique. Dans le film Persepolis, Satrapi et Paronnaud ont supprimé des scènes, des détails et même des personnages trouvés dans les albums en vue de respecter la durée moyenne d'un film animé. De plus, les deux réalisateurs du film ont ajouté des passages introuvables dans les albums en prenant en considération que les passages ajoutés ou supprimés doivent être soigneusement choisis pour ne pas perturber l'histoire originale.

Persepolis a eu le plus grand succès de la bande dessinée européenne des années 2000 grâce à une auteure et dessinatrice bien douée. ${ }^{\mathrm{i}} \mathrm{Vu}$ le succès remarquable de Persepolis comme BD puis comme film, nous avons choisi cette œuvre pour résoudre notre problématique. Le film Persepolis est l'investissement du succès de la BD préalable. À notre sens, le but de Marjane Satrapi, dans la réalisation du film, est de montrer une image plus réaliste de son histoire par l'animation. Certainement, il y a un sens supplémentaire que le film nous donne à sentir. De fait, le film ajoute une valeur documentaire à la vie du peuple iranien dans ce temps-là, même si la $\mathrm{BD}$ constitue la seule référence pour le film mais nous devons déduire, à travers notre recherche qu'il s'agit de la représentation la plus productive du sens, soit la BD, soit le film.

Notre hypothèse de départ est la suivante : la lecture de l'image doit se faire en parallèle avec la lecture du discours verbal pour 
comprendre le sens d'une image soulignée ou complétée par un texte ou d'un texte illustré par une image. En effet, nous mettrons l'accent sur la relation image/discours qui joue un rôle indispensable dans la compréhension des intentions et des allusions de Marjane Satrapi dans son autobiographie imagée.

Nous ne pouvons atteindre le sens de l'histoire sans relier le discours à l'image. Surtout que l'auteure n'écrit pas toujours ce qu'elle veut dire mais elle le dessine. Dans cette recherche, nous poserons des questions pour en trouver les réponses qui seront nos résultats finals :

- Est-ce que l'analyse de l'image serait subjective ou objective ?

- Est-ce que l'image pourrait faire une confusion dans l'interprétation du texte?

De plus, nous ferons une comparaison entre les deux moyens de présentation du même récit (BD et cinéma) parce que l'analyse diffère certainement entre les albums de la BD et le film, surtout quand les dessins sont réalisés en noir et blanc et les détails de l'atmosphère ne sont pas définitivement clairs dans l'image. 


\section{Analyse comparative des albums de la BD Persepolis et de son adaptation filmique}

Plus souvent, quand les lecteurs, les critiques, les chercheurs regardent un film basé sur une source littéraire, une de leurs premières pensées est de faire une comparaison entre le film et la source originale. Particulièrement, quand il y a un défi de réaliser un film extraordinaire qui diffuse des idées censurées.

Quand nous parlons de l'adaptation du point de vue du système d'interpréter ou de déchiffrer le sens de la source originale (la BD) par un autre système de signes (le film cinématographique), nous observons que le processus de la transmission de la BD au cinéma semble presque le concept de la traduction qui doit maintenir les mêmes idées, valeurs et sens lorsque nous expliquons, discutons ou traitons un thème écrit par une langue à travers une autre. C'est pourquoi la comparaison entre les deux supports de l'adaptation est également connue par les auteurs qui font les traductions d'une langue à une autre et par les cinéastes qui produisent des adaptations littéraires.

En général, les critiques déclarent qu'une traduction n'est jamais égale à la source originale parce que les expressions des langues diffèrent les unes des autres et le sens sera obligatoirement changé. Dans la plupart du temps, les commentaires favorisent la version originale qui transmet énormément l'esprit littéraire et la puissance dynamique de l'originalité. Cependant, l'adaptation filmique de Persepolis est un cas différent car Marjane Satrapi vise à rapprocher le sens de la $\mathrm{BD}$ à travers son adaptation filmique qui a connue beaucoup de louange de la part des spectateurs et des critiques à la fois. 
En effet, tous les styles divers des adaptations sont néanmoins liés par les mêmes normes de base de la source originale. Pourtant, la performance cinématographique est forcément différente de la représentation de la bande dessinée car les deux supports s'appuient sur des répertoires différents. La question qui se pose perpétuellement est de savoir s'il y a des différences essentielles et considérables entre les deux formes qui donnent lieu aux différents modèles et normes artistiques.

Dans le processus de l'adaptation filmique, les cinéastes ne peuvent pas ignorer ni l'arrière-plan de la culture cible, ni l'interaction de l'audience avec la source originale. Les métaphores culturelles et les descriptions sont parfois difficiles à transférer à l'écran, et elles subissent des changements importants lors de la transformation dans un scénario menant à la production d'un film.

Dans le cinéma, les métaphores se transforment en images plus compréhensibles, les expressions idiomatiques des pages sont remplacées par des expressions explicites de la vie réelle par des personnages réels, les émotions sont expliquées ou transposées dans le but de les rendre plus touchables et plus accessibles au public. Pour cela, nous devons mettre l'accent sur le côté sentimental dans notre comparaison.

En outre, le film possède une relation plus intéressante avec la réalité par le langage qu'il utilise pour transférer les significations dans un temps réel. Cela peut expliquer pourquoi de telles adaptations sont devenues plus proches au public que les ouvrages originaux. Si nous voulons mieux comprendre l'adaptation cinématographique, nous devons la reconnaître comme un sousgenre de cinéma très distinct par la comparaison avec la source 
originale. Par ailleurs, l'adaptation a des caractéristiques spécifiques de l'esthétique grâce à l'interprétation universelle.

Même dans les films adaptés, le cinéma existe en tant qu'un art parce qu'il crée quelque chose de nouveau d'une manière authentique et non parce qu'il s'adapte simplement à un modèle préexistant. En tout cas, les adaptations cinématographiques visent à moderniser fréquemment la source originale à tous les niveaux parce que le processus du transfert vers un autre support encourage la récréation et la novation.

\section{Comparaison générale entre la BD et le film Persepolis}

Avant de commencer notre comparaison entre les deux supports, il faut mentionner que nous sommes devant la même histoire soit dans les albums de la BD, soit dans le film Persepolis, mais le traitement de chacun des deux supports artistiques est tout à fait distinct et différent car la cinématographie a ses propres traits caractéristiques à appliquer l'industrie de l'adaptation cinématographique ayant des conditions précises à suivre pour réaliser le produit filmique final. Au moment où, la bande dessinée se caractérise par la linéarité, le cinéma se caractérise par l'allusion.

Après avoir étudié la BD Persepolis puis son adaptation cinématographique, il sera plus accessible de mettre l'accent sur les éléments communs puis les techniques différentes dans les deux supports que Marjane Satrapi a ajoutés ou supprimés dans la BD et son adaptation filmique pour raconter son expérience personnelle et parfois sa souffrance à son public dans son autobiographie reconnue dans une BD et un film. 
Passons maintenant à exposer, dans les pages suivantes, les points communs et les points différents entre les supports que nous avons étudiés pendant notre recherche de l'autobiographie Persepolis :

\section{A- Les points communs entre la BD et le film :-}

Au cours de notre analyse du film Persepolis, nous avons remarqué plusieurs points communs avec la BD. Sans doute, ces points organisent le travail de la production de l'adaptation puisque Marjane Satrapi, l'auteure et la dessinatrice de la même histoire en $\mathrm{BD}$, est particulièrement la réalisatrice du film cinématographique.

\section{1- La simplicité dans le dessin}

Certes, nous remarquons bien que la dessinatrice Marjane Satrapi aime toujours les lignes simples qui ne contiennent pas de détails dans ses dessins soit dans la BD, soit dans le film. Bien que les images de la BD soient différentes dans le cadrage de celles du film, toutes les deux expriment quelquefois la même scène avec le même discours. Par ailleurs, il nous faut noter que la qualité des images filmiques est plus claire que celle des images de la bande dessinée grâce aux techniques avancées de la réalisation cinématographique moderne.

\section{2- La conformité entre l'image et le discours}

Dans les deux supports, nous trouvons une convention considérable, dans la plupart des cas, entre le discours et l'image de la même scène. Quelquefois, nous remarquons la même scène avec le même discours dans la BD et dans le film aussi.

Quand la mère voulait exprimer sa grande peur à sa petite fille, nous voyons que Marjane Satrapi fonctionnent parallèlement les mêmes discours et images dans les deux supports. De plus, nous 
remarquons clairement qu'il y a une redondance majeure entre l'image et le discours pour dessiner la même idée parce que toutes les images de cette scène décrivent habilement la peur de la mère à travers les expressions du visage et les gestes corporelles à côté du discours.

\section{3- La force de la langue}

Dans les deux supports, la langue est forcément indispensable surtout à travers les paroles de la narratrice parce que l'image ne peut pas parfois exprimer ce que la langue peut largement dire. En effet, la langue a un effet magique et efficace concernant les sentiments, les idées et les détails qui ne peuvent être décrits par les images seulement.

$\sqrt{ }$ Par ailleurs, nous trouvons que la langue utilisée a recours aux phrases à la forme négative puisque rien ne peut habilement exprimer la négation que la langue. En voici des exemples :

\section{Dans la BD}

$\underline{\text { Le premier album }}$

La narratrice dit: "Je ne savais pas trop quoi penser au foulard. Moi, j'étais très croyante mais moi et mes parents ensemble étions très modernes et avant-garde."

$\underline{\text { Le deuxième album }}$

La narratrice dit: "Un grand rêve venait de s'écrouler. Jamais je n'aurais le droit d'aller aux États-Unis."

$\underline{\text { Le troisième album }}$ 
Marjane dit à ses amis : "L'existence n'est pas absurde. Il y a des gens qui donnent leur vie pour des valeurs comme la liberté."

Le quatrième album

Marjane dit au psychiatre : "J'ai honte de n'avoir rien fait de ma vie. Heureusement, personne n'est au courant des détails et pour cause! Je ne leur raconte rien."

\section{Dans le film}

Marjane dit à ses amis : "L'existence n'est pas absurde. Tu sais qu'il y a des gens qui donnent leur vie pour la liberté." (48:16)

La narratrice dit: "L'État n'avait bien entendu aucune raison de s'inquiéter." (51:50)

La narratrice dit : "Rien n'avait changé. Pourtant, je le savais au fond de moi, plus rien ne sera comme avant." (01:01:15)

La narratrice dit : "Le gouvernement avait prisonniers tellement de lycéens et d'étudiants que nous n'osions plus parler politique." (01:11:40)

$\sqrt{ }$ De plus, nous trouvons des phrases interrogatives écrites dans le dessin des bulles de la BD et dites dans le discours du film quand un des personnages du récit voudrait poser une question et l'image ne peut jamais la poser. En voici des exemples :

\section{Dans la BD}

Le premier album

Quand la petite Marji regarde les anciennes photos de la famille, elle observe l'absence de son grand-père et elle demande à sa grandmère : "Pourquoi n'y a pas papi ? Il était en prison?" 


\section{Le deuxième album}

Le père demande à l'homme qui fabrique les passeports : "Il te faut combien de temps pour faire un passeport?"

Le troisième album

Quand Marjane devient déçue, elle se demande : "Où étaient mes parents pour me prendre dans leurs bras ?"

$\underline{\text { Le quatrième album }}$

Après le retour de Marjane, son père lui demande : "Comment tu trouves Téhéran ?"

\section{Dans le film}

Quand la petite Marji voit une de leurs proches à l'aéroport dès son arrivée à l'Iran de la France, elle lui demande curieusement : "C'est comment Paris ? Et la tour Eiffel, c'est comment ?" (03:00)

Quand la petite Marji veut être le dernier prophète, elle impose des lois et des règles dans son livre sacré. Pour cela, la grand-mère demande à sa petite-fille : "Dis-moi comment tu vas faire pour que les vieilles ne souffrent plus?" (04:45)

Quand la maîtresse défend le régime islamiste, Marjane lui demande : "Comment osez-vous de nous mentir comme ça ?" (38:40)

Bref, nous remarquons que l'image ne peut pas être suffisante pour accomplir le sens dans le récit Persepolis soit dans la BD, soit dans le film. Par conséquence, le fonctionnement de la langue est indispensable dans la narration des événements. 


\section{الجمعية المصرية للقر اعة والمعرفة عضو الجمعية الدولية للمعرفة}

\section{4- La force de l'image}

Comme l'image est le thème principal de notre travail, nous devons mettre en relief le rôle considérable qu'elle joue parallèlement dans les quatre albums de la BD et dans l'adaptation filmique. Dans les deux supports, il ne faut pas négliger la force artistique de l'image comme moyen sensible de l'expression du sens. Nous remarquons bien que les images de la dessinatrice Marjane Satrapi dépassent souvent le discours pour déclarer un sentiment caché ou pour dire plus que les mots.

\section{B- Les points différents entre la BD et le film :-}

Pour que Marjane Satrapi et Vincent Paronnaud réalisent bien leur film Persepolis en adaptant la même histoire de la BD, ils ont dû respecter la nature et les techniques de l'industrie cinématographique en appliquant quelques opérations essentielles qui ne peuvent pas être existées dans la BD :

\section{1- La complexification}

Pour faire une adaptation filmique, la complexification apparait dans le comment d'exposer bien un récit pendant une heure et demie au lieu de la lecture d'une longue série des pages. Principalement, le support cinématographique compte sur le langage allusif. Quand nous regardons un tel film, nous comprenons quelquefois le sens d'une manière indirecte. Dans le film Persepolis, les deux réalisateurs ont souvent recours à la complexification plus que dans la BD.

\section{2- La suppression}

Comme l'histoire de la BD se compose de quatre albums, les réalisateurs du film sont obligés à supprimer quelques détails par 
l'ellipse ou par le montage pour achever leur produit filmique. En conséquence, nous ne trouvons pas certaines situations de l'autobiographie de Marjane dans le film, comme :

- Le premier album de la BD met l'accent sur l'injustice sociale en Iran à travers la relation amoureuse de la servante Mehri qui adore le voisin de Satrapi malgré le décalage entre leurs classes sociales.

- Le deuxième album de la BD raconte le voyage des parents pour la Turquie pour qu'ils puissent acheter des marchandises importées qui ne se trouvent plus en Iran pendant la guerre Irakoiranienne.

- Le troisième album de la BD raconte l'installation de Marjane chez son amie Julie en Autriche après qu'elle quitte la pension des bonnes sœurs.

- Le troisième album de la BD raconte la visite de la mère de Marjane en Autriche pour voir sa fille.

- Le troisième album de la BD décrit l'état de la monotonie et de la solitude dont Marjane souffre en Autriche, pour cela elle a recours aux exercices de Yoga pour perdre du temps.

- Le quatrième album de la BD raconte la candidature de Marjane à la faculté des arts avec Réza.

- Le quatrième album de la BD raconte le concours artistique auquel Marjane et son mari Réza participent en Iran.

\section{3- La substitution}

Bien que Marjane Satrapi présente son autobiographie dans la $\mathrm{BD}$, elle ne suit pas le même ordre des événements dans le film.

L'histoire débute en 1979 dans la BD mais elle débute en 1978 dans 
le film. De plus, Marjane rencontre Réza en 1989 dans la BD mais elle le rencontre en 1992 dans le film. En outre, Marjane se marie avec Réza en 1991 dans la BD mais elle se marie avec lui en 1993 dans le film, ... etc.

\begin{tabular}{|c|c|c|}
\hline & Dans la BD & Dans le film \\
\hline Le début de l'histoire & 1979 & 1978 \\
\hline Le rencontre avec Réza & 1989 & 1992 \\
\hline Le mariage avec Réza & 1991 & 1993 \\
\hline
\end{tabular}

\section{4- La voix-off}

La voix-off est le contraire de la voix-in. C'est une voix enregistrée par un narrateur ou par un des personnages du film pour commenter les événements exposés. La voix off est considérée un des éléments essentiels du support cinématographique qui est utilisée pour faire réussir toutes les opérations précédentes. À travers la voix off, les cinéastes peuvent bien faire la suppression, l'enchevêtrement, la complexification, la substitution, la condensation, ... etc.

Dans le film Persepolis, la voix off de la narratrice est celle de Marjane elle-même. La voix off est émotionnelle, expressive et communicative. À cause de la durée courte du film, les paroles de la narratrice dans la BD sont plus que celles dans le film. Dans notre analyse, nous faisons la différence entre les paroles de Marjane comme la narratrice et ses paroles comme un personnage dans le film. 


\section{5- La condensation}

Pour respecter la durée ordinaire du film, la condensation joue un rôle remarquable en vue de résumer quelques détails en une seule scène ou parfois en une seule phrase. C'est pourquoi, les quatre albums de la BD Persepolis sont plus riches en détails que le film du même récit. Bien que Marjane Satrapi explique minutieusement des situations intimes dans les albums, elle les résume brièvement dans son film, comme :

- La BD montre en détails toutes les places où Marjane habite pendant sa longue série des déménagements en Autriche. Cependant, la narratrice compte seulement sur la voix-off pour résumer les déménagements de Marjane en Autriche dans un petit passage dans le film.

- La BD montre toute la visite de Marjane chez son ami Kia et nous y lisons tout leur long dialogue. Cependant, le film mentionne seulement que Marjane a visité son ancien ami Kia.

- La BD déclare le rôle de Roxana l'amie iranienne qui aime organiser des fêtes chez laquelle Marjane a rencontré Réza pour la première fois. Cependant, le film cite seulement que Marjane a assisté à une fête chez une de ses amies.

- La BD décrit minutieusement la première rencontre entre Marjane et Réza chez Roxana et nous y lisons tout leur long dialogue. Cependant, le film exprime la connaissance entre Marjane et Réza à travers une scène romantique muette dans une fête.

- La BD montre toutes les préparations du mariage de Marjane et Réza depuis la première rencontre de Réza avec son père qui contient beaucoup de conventions, conditions et détails jusqu'aux habitudes iraniennes de la célébration du mariage. Cependant, le 


\section{ILA الجمعية المصرية للقراءة والمعرفة عضو الجمعية الدولية للمعرفة}

film montre seulement des photos de leur noce pour nous annoncer leur mariage.

\section{6- L'enchevêtrement}

En fait, l'enchevêtrement est tout simplement la non-linéarité. Quand le lecteur lit les albums de la BD Persepolis, il poursuit linéairement les planches des dessins pour comprendre l'histoire. Mais selon la nature de l'industrie cinématographique, les metteurs en scène du film Persepolis doivent délinéariser le récit adapté pour achever un bon film. Raison pour laquelle, le spectateur reçoit inconsciemment le moral du récit en regardant le film sans exercer aucun effort.

\section{- Conclusion}

Par notre comparaison, nous avons remarqué que l'adaptation cinématographique de Persepolis est plus vague que la BD, particulièrement en ce qui concerne les références temporelles. Bien que chaque support ait une spécificité inhérente à sa nature, Marjane Satrapi déclare très haut dans les deux supports artistiques différents (la BD et son adaptation filmique) que l'être humain est le même partout et il a les mêmes rêves. Selon nous, le récit de Persepolis est une leçon d'humanité et de courage pour toutes les femmes.

Dans ce travail, les résultats de l'analyse confirment l'hypothèse de départ qui impose la fatalité de comprendre le discours pour comprendre l'image afin d'atteindre le sens. Dans Persepolis, l'image et le discours ont une relation réciproque dans la compréhension du sens, et par conséquent les deux se complètent toujours dans la BD et dans le film. En général, Marjane Satrapi dessine ce qu'elle veut dire et vice-versa. 
D'ailleurs, l'image nous aide beaucoup à interpréter correctement les intentions et les allusions de l'auteure dans le discours parce qu'elle a recours souvent à fonctionner ses dessins pour exprimer ses idées et ses sentiments par les gestes, les mimiques, les expressions du visage, le langage du corps, ... etc. C'est pourquoi nous ne trouvons aucune confusion dans le déchiffrement du sens des images.

De plus, notre recherche dévoile que les adaptations cinématographiques doivent être vues, étudiées et analysées sémiotiquement comme un produit hybride résultant de la fusion de plusieurs auteurs, cinéastes et cultures parce qu'il s'agit d'un processus dynamique et interactif entre deux supports artistiques différents.

Finalement, Persepolis est un récit en BD puis un film en noir et blanc mais il est haut en couleurs sentimentales. Nous voudrions exprimer notre conviction du brillant avenir de la BD hypermédiatique. À partir de notre recherche, nous observons un grand enthousiasme et une soif de continuer les expérimentations artistiques en $\mathrm{BD}$, soit littérairement, soit cinématographiquement, qui seront menées à des recherches très intéressantes. Notons qu'une finalité peut en cacher un début !

\section{- Bibliographie}

\section{I- Corpus De L'étude}

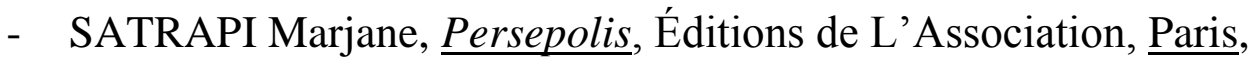
France.

1. Tome 1, 2000 (ISBN 2-84414-058-0) 
2. Tome 2, 2001 (ISBN 2-84414-079-3)

3. Tome 3, 2002 (ISBN 2-84414-104-8)

4. Tome 4, 2003 (ISBN 2-84414-137-4)

- SATRAPI Marjane avec PARONNAUD Vincent (2007), Film de Persepolis (d'après l'œuvre originale de Marjane Satrapi), produit par Marc-Antoine Robert et Xavier Rigault (2.4.7. Films), Paris.

\section{II- Ouvrages Consacrés À La Sémiotique}

- BARTHES Roland (1969), Éléments de sémiologie, Gonthier, Paris.

- BERTRAND Denis (2000), Précis de sémiotique littéraire, Nathan, Paris.

- CHABROL Claude et ALEXANDRESCU Sorin (1973), Sémiotique narrative et textuelle, Larousse, Paris.

- COURTÉS Joseph (1991), Analyse sémiotique du discours, Hachette, Paris.

- COURTÉS Joseph (2003), La sémiotique du langage, Nathan, Paris.

- ECO Umberto (1988), Sémiotique et philosophie du langage, Presses Universitaires de France, Paris.

- ECO Umberto (1980), Le signe, Livre de poche, Paris.

- EVERAERT-DESMEDT Nicole (2007), Sémiotique du récit, De Boeck, 4éd, Bruxelles.

- FISETTE Jean (1993), Introduction à la sémiotique de Peirce, XYZ éditeur, Montréal. 
- FONTANILlE Jacques (2003), Sémiotique du discours, PULIM (coll. Nouveaux Actes Sémiotiques), Limoges.

- FONTANILLE Jacques (1999), Sémiotique et littérature (Essais de méthode), Presses Universitaires, Limoges.

- GREIMAS Algirdas Julien et COURTÉS Joseph (1979), Sémiotique (Dictionnaire raisonné de la théorie du langage), tome I, Hachette, Paris.

- GREIMAS Algirdas Julien et COURTÉS Joseph (1986), Sémiotique (Dictionnaire raisonné de la théorie du langage), tome II, Hachette, Paris.

- GREIMAS Algirdas Julien et COURTÉS Joseph (1993), Dictionnaire raisonné de la théorie du langage, Hachette, Paris.

- GREIMAS Algirdas Julien (1970), Du sens I, Seuil, Paris.

- GREIMAS Algirdas Julien (1983), Du sens II, Seuil, Paris.

- GREIMAS Algirdas Julien (1976), Introduction à la sémiotique narrative et discursive, Hachette, Paris.

- GREIMAS Algirdas Julien (1986), Sémantique structurale, Presses Universitaires de France, Paris.

- MILLOGO Louis (2009), Introduction à la lecture sémiotique, Harmattan, Paris.

\section{III- Ouvrages Consacrés Aux Bandes Dessinées}


- ALBERELLI Christian (1989), Bande dessinée et littérature graphique, CRDP, Grenoble.

- BARON-CARVAIS Annie (1994), La bande dessinée, Collection "Que sais-je ?", Presses Universitaires de France, Paris.

- BELLEFROID Thierry (2005), Les Éditeurs de bande dessinée, NIFFLE, Bruxelles.

- BOUGNOUX Daniel (1991), La communication par la bande, La Découverte, Paris.

- CONVARD Didier, SERGE Saint-Michel et GOSCINNY René (1972), Le français et la bande dessinée, Nathan, Paris.

- GAUMER Patrick (1997), La bande dessinée en France, Association française d'action artistique, Paris.

- GROENSTEEN Thierry (1999), Système de la bande dessinée, PUF, Paris.

- JOLY Martine (1993), Introduction à l'analyse de l'image, Nathan, Paris.

- MASSON Pierre (1985), Lire la bande dessinée, Presses universitaires de Lyon, Lyon.

- RENARD Jean-Bruno (1985), La Bande dessinée, Segers, Paris.

- VETTRAINO-SOULARD Marie-Claude (1993), Lire une image, Armand Colin, Paris.

IV- Ouvrages Consacrés Au Cinéma 
- BAZIN André (1958), Qu'est-ce que le cinéma ?, Collection "Septième Art", Tome I, Ontologie et langage, Cerf, Paris.

- BAZIN André (1959), Qu'est-ce que le cinéma ?, Collection "Septième Art", Tome II, Le cinéma et les autres arts, Cerf, Paris.

- BAZIN André (1961), Qu'est-ce que le cinéma ?, Collection "Septième Art", Tome III, Cinéma et sociologie, Cerf, Paris.

- BAZIN André (1962), Qu'est-ce que le cinéma ?, Collection "Septième Art", Tome IV, Une esthétique de la Réalité : le néoréalisme, Cerf, Paris.

- BAZIN André (1987), Qu'est-ce que le cinéma ? Collection dirigée par Guy Hennebelle, Cerf, Paris.

- GARDIES René (2007), Comprendre le cinéma et les images, Armand Colin, Paris.

- GAUDREAULT André (1999), Du littéraire au filmique, Éditions Nota Bene, Montréal.

- JOURNOT Marie-Thérèse (2003), Le vocabulaire du cinéma, Nathan, Paris.

- MERLEAU-PONTY Maurice (2009), Le cinéma et la nouvelle psychologie, Gallimard, Paris.

- METZ Christian (1977), Essais sémiotiques, Klincksieck, Paris.

- METZ Christian (1971), Essais sur la signification au cinéma, Tome I, Klincksieck, Paris. 
- METZ Christian (1972), Essais sur la signification au cinéma, Tome II, Klincksieck, Paris.

- METZ Christian (1971), Langage et Cinéma, Larousse, Paris.

- METZ Christian (1984), Le signifiant imaginaire : psychanalyse et cinéma, Bourgeois, U.G.E., Paris.

- PINEL Vincent (1993), Techniques du cinéma, P.U.F., Collection "Que sais-je ?" Paris.

${ }^{i}$ En 2007, Vincent Paronnaud reçoit avec Marjane Satrapi le Prix du Jury du Festival de Cannes pour Persepolis qui gagne également deux Césars l'année suivante : ceux du meilleur premier film et de la meilleure adaptation. Le film a également été nommé aux Oscars 2008 dans la catégorie Meilleur film d'animation. https://fr.wikipedia.org/wiki/Winshluss consulté le 18/1/2017 